\title{
4. Case studies of universities in Europe
}

\subsection{RESEARCH DESIGN AND SELECTED UNIVERSITIES}

In our general model of an RII system and its derivative RII analytical framework (presented in Part I of this book as Figures 1.1, 2.1 and 3.1) we try to 'square this circle' in an abstract manner. Moving into the realm of empirical information gathering and obtaining evidence, we need to recognise that each European university, embedded in its local region, defines its own unique system of assets, resources, opportunities and obstacles. How to capture such diversity, dynamics, and uniqueness? Not only with regard to the RII potential and performance of each university, but also regarding background information and the local socio-economic circumstances in which it operates. Capturing the complexity of those RII systems and getting to grips with relevant attributes, such as RII pathways, begs several questions. To what degree does the model represent RII realities within universities? Can the RII analytical framework deliver? Is it sufficiently versatile to collect a wide range of relevant information?

The only way to convincingly answer the above questions is to put the analytical framework to the test. We applied an analytical framework consisting of the four RII portfolio domains and main components of the RII analytical framework (see Box 4.1).

\section{BOX 4.1 DESCRIPTIVE FRAMEWORK FOR THE SELF-APPRAISAL REPORTS: RII DOMAINS AND MODEL COMPONENTS}

RII domains:
A. Regional orientation, strategic development and knowledge infrastructure;
B. Education and human resources development;
C. Research, technological development and knowledge transfer;
D. Support to enterprise development and entrepreneurship. 
RII model components:

1. Needs, problems and issues articulated by external parties in the local or regional environment;

2. Organisational goals, internal driving forces and/or motivational factors within the university;

3. Resources, general facilities and/or incentive systems within the university;

4. Pathways, outreach and engagement connections between the university and outside agents;

5. Delivered impacts (RIIs) by the university;

6. Relationships between RII initiatives, capacities, and competences within the university and those in its home city or region.

Our information was extracted from two sources:

- A mail questionnaire we distributed across 20 research-active universities and 13 countries in the EU28. We asked about their RII capacity, capabilities, and challenges. The survey's seven questions address the RII model components 2 and 3 listed in Box 4.1. Section 4.2 describes this questionnaire in more detail and presents the survey's main findings.

- We enrolled a selection of universities in Europe to conduct their own RII self-appraisal reporting on their RII profile. Each were invited by the European Commission's DG Joint Research Centre (JRC) to participate in this pilot study and submit a 'narrative with numbers' report, according to specific instructions described in Appendix A, that summarises relevant information on their past RII achievements, current RII potential as well as their vision and plans for the near future. These cases studies, conducted in 2018, were primarily designed as a mutual learning platform and to help us gauge the feasibility and utility of the framework. ${ }^{1}$

The questionnaire and case study approach provide a rich view of RII-relevant issues from different angles. Although the collected information is incomplete, and possibly biased, the evidence is valuable for testing the analytical strength of a structured 'narrative with numbers' approach and the way it could be implemented as an RII self-appraisal reporting tool.

The universities that took part in this study were approached after presentations of the RII framework at events and meetings organised by relevant

1 The universities may have used this opportunity, without our knowledge, to engage in more detailed assessments for their own internal purposes. 
working groups of the European University Association (EUA), ${ }^{2}$ CESAER $^{3}$ and ECIU. ${ }^{4}$ The participating universities were brought together with regional government agencies, European Commission officials and representatives from the EUA, CESAER and ECIU in four events: two in Brussels, one organised by Aalborg University and another organised by the University of the Basque Country. These events facilitated mutual learning in the drafting of the case studies and a further reflection on the RII analytical framework. Universities were also supported by JRC researchers through the review of (draft versions of) submitted case study reports.

Their participation in those case studies was voluntary. This imposed some constraints on attaining an optimal geographical coverage. For example, no universities are included from some large European countries such as Germany or France. The reason for this is that either no interested university was identified among the membership of the university associations, or because the participating universities withdrew at a later stage in the development of their case studies. In spite of these constraints the sample of participating universities includes universities from the South, East, North and West of Europe; and from regions at different levels of economic development and absorptive capacity. The sample also includes different types of universities: $^{5}$ comprehensive research universities such as the Catholic University of Leuven, Leiden University and Bologna University; leading technical research universities such as the Technical Universities of Turin, Milan, Catalunya, Delft University and the University of Strathclyde; young innovative universities such as Aalborg and Aalto universities and universities with an explicit regional orientation such as Rovira i Virgili University, the University of the

2 The European University Association (EUA) represents more than 800 universities and national rectors' conferences in 48 European countries.

3 The Conference of European Schools for Advanced Engineering Education and Research (CESAER) is a research stakeholder organisation comprising 53 specialised and comprehensive universities of science and technology in European countries (www .cesaer.org).

4 The European Consortium of Innovative Universities (ECIU) is a network of 14 relatively young universities who are strongly committed to the encouragement of innovation and entrepreneurship, with close ties to industry in their region and a focus on innovative teaching approaches.

5 The classification of university types is fluid, in part because of the current state of conceptual development in the studies of these organisations. For example, there is no agreed upon definition of an entrepreneurial university: the Catholic University of Leuven could be classified as both a comprehensive university; a technical university as a member of CESAER given its strong engineering capabilities; a 'glocal' university with a strong impact on both the high tech region it operates in as well as being very successful at the global stage; while it also incorporates some elements of what could be understood as an entrepreneurial university. 
Table 4.1 European research-active universities participating in the case studies

\begin{tabular}{|c|c|c|c|c|}
\hline & & $\begin{array}{r}\text { ISCED 5-7 } \\
\text { graduates } \\
{\text { (count) })^{1}}^{2}\end{array}$ & $\begin{array}{r}\text { Research } \\
\text { publication } \\
\text { output } \\
(\text { count })^{2}\end{array}$ & $\begin{array}{c}\text { Regional } \\
\text { Innovation } \\
\text { category }\end{array}$ \\
\hline University of Bologna & Italy & 18833 & 16757 & Moderate \\
\hline Catholic University of Leuven & Belgium & 14563 & 24823 & Strong \\
\hline University of Warsaw & Poland & 13255 & 6703 & Moderate \\
\hline Technical University of Milan & Italy & 11796 & 7864 & Moderate \\
\hline University of the Basque Country & Spain & 7976 & 9104 & Moderate \\
\hline Norwegian Univ. of Sci. and Techn. & Norway & 7847 & 10055 & Leader \\
\hline Leiden University & Netherlands & 7495 & 16442 & Leader \\
\hline University of Strathclyde & United Kingdom & 6885 & 4952 & Strong \\
\hline Technical University of Turin & Italy & 6466 & 4691 & Moderate \\
\hline Eötvös Loránd University & Hungary & 6442 & 3666 & Moderate \\
\hline Aalborg University & Denmark & 6169 & 6973 & Strong \\
\hline Technical University of Catalunya & Spain & 5818 & 6627 & Moderate \\
\hline Delft University of Technology & Netherlands & 5203 & 10797 & Leader \\
\hline Aalto University & Finland & 3968 & 7351 & Leader \\
\hline University of Trieste & Italy & 3373 & 4731 & Strong \\
\hline University of Aveiro & Portugal & 2924 & 6265 & Moderate \\
\hline Rovira i Virgili University & Spain & 2751 & 3677 & Moderate \\
\hline University of Stavanger & Norway & 2474 & 1911 & Strong \\
\hline Kaunas University of Technology & Lithuania & 2378 & 1413 & Moderate \\
\hline University of Ruse & Bulgaria & 1844 & 44 & Modest \\
\hline
\end{tabular}

Sources: ${ }^{1}$ ETER database (August 2019). ISCED 5-7: undergraduate students ('Short-cycle tertiary education', 'Bachelor or equivalent', 'Master or equivalent'). Data reference year is 2016; ${ }^{2} \mathrm{Web}$ of Science database (Leiden University); U-Multirank 2018 data (publication years 2013-2016); ${ }^{3}$ Regional Innovation Scoreboard 2019; 'Performance Group' data (https://ec .europa.eu/growth/sites/growth/files/ris2019.pdf).

Basque Country, University of Trieste and the University of Ruse. A full overview of the participating universities is provided in Table 4.1.

As a whole, these two information sources provided a valuable overview of RII ambitions, competences, activities and achievements. Chapters 5 to 8 describe and summarise those case study findings according to the four RII portfolio domains. Each domain emphasises different RII model components. For example, the domain 'Regional orientation, strategic development and knowledge infrastructure' is mainly devoted to components 1, 2 and 6, while 'Education and human resources development' focuses on components 3 and 
4 in the RII model. The various components are not always easily recognisable in the case studies.

The total set of 20 presents an interesting and illustrative cross-section of universities in Europe. It includes both broad 'comprehensive' ones, as well as those more 'specialised' in particular disciplinary areas (several are universities of technology). Several of the 20 are among the most high-profile research-active universities worldwide in terms of their international reputation and research performance. Many are one of the largest employers in their home towns. There are both medium and large-sized universities. The largest in terms of 'total graduates' (bachelor, master and doctoral students), the University of Bologna, is ten times larger than the University of Ruse. As for doctoral graduates, the Catholic University of Leuven is 20 times larger than Kaunas University of Technology in Lithuania and the University of Stavanger. The number of research publications in international scientific journals is a crude proxy of scientific research activities. The large research-active universities in Western Europe produce more publications than those in Eastern Europe, in order of magnitude; in the case of the University of Ruse, two orders of magnitude. These publication output counts are determined by many factors, apart from the ability to undertake international-level research. Nonetheless, the observed differences in terms of research capabilities are large and will affect their RII potential and RII pathways.

Given their interest to join our pilot study, our selection of universities is more than likely to represent those organisations that value or prioritise their regional engagement activities. As such, they are more likely to have developed successful RII strategies, capacities, and competences. If we consider these RII-active universities to be 'at the frontier' of these developments in Europe, it is imperative to consider their regional differences. As shown in Table 4.1, the innovation performance level of those regions, according to 2019 editions of Europe's Regional Innovation Scoreboard, varies from 'Leader' (four universities) to 'Modest' (one university). The other 15 universities are located in either a 'Strong' or 'Moderate' performing region. One cannot isolate a university's RII potential from its region's innovation performance. They are usually strongly connected, and probably interdependent.

The imposed constraints on the size of the self-appraisal reports (see Appendix A) prevented universities from providing full-scale, in-depth overviews of their RII profile. Nor was the information provided by the universities verified or validated by an external party. Given the limitations in their brief, it is not very surprising to see that their case study material tends to emphasise their achievements rather than presenting a critical review of their RII potential or performance, and provide no details on causal relationships within RII processes nor explanations for RII outcomes. 
Featuring selected information from the case studies, Chapters 5 to 8 illustrate the breadth and depth of the information provided by the universities as well as the diverse nature of their RII portfolios and profiles. Some universities provided extensive and well-structured self-appraisal reports. Edited and shortened versions of five case studies are included in Appendixes B-F: Leuven University, Aalborg University, Technical University of Turin, Rovira i Virgili University, and Warsaw University. Two of these universities are from 'Strong' innovative regions and three from regions with a 'Moderate' performance.

Nonetheless, these self-appraisal reporting case studies present many interesting examples of the RII 'narrative with numbers' approach. They illustrate the many faces of RII, reveal a range of available in-house information within universities, and a variety of indicators they apply to substantiate their performance. The submitted empirical information also offers a glimpse of RII-relevant 'good practices' across European research-active universities. It also provides clues as to the feasibility of applying the RII analytical framework in practice, and the kind of RII-relevant facts that universities are able to supply, within a reasonable time-span, either for internal or external assessment.

\subsection{SURVEY ON RII POTENTIAL AND ORGANISATIONAL CHALLENGES}

Regional engagement is a necessary condition for any kind of RII potential and performance, though it may not be a sufficient condition to generate RII outcomes. To attain a better understanding of this engagement, our survey was designed to collect background information from the 20 universities on their RII performance profile. Driven by our RII analytical framework depicted in Figure 2.1 (section 2.1), our goal was to unearth information and impressions about their goals and motivation to engage in RII, the available in-house organisational resources, and whether or not they have implemented incentive systems to encourage regional engagement. We were also interested to learn in which particular RII portfolio domains they experience problems in regional engagement activities or obstacles to achieve their RII objectives. We were looking for new insights into their current RII-relevant processes and practices. Our aim was to detect general patterns that will help better understand critical factors in activating RII potential.

Ultimately, 12 universities returned our questionnaire: Aalborg University, Aalto University, Catholic University of Leuven, Kaunas University of Technology, Rovira i Virgili University, Technical University of Catalunya, Technical University of Turin, University of Aveiro, University of the Basque Country, University of Ruse, University of Stavanger, and University of 
Warsaw. Collectively, they represent an interesting geographical cross-section of regionally active universities in northern, southern, eastern and western Europe. The survey findings are not representative for European research universities in general, but provide a general impression of what is happening in regionally engaged research-active universities.

In response to our first question, the large majority of those universities have adopted explicit strategies dedicated to 'regional orientation and engagement'; only two universities indicate that their regional priorities are implicit in the overall strategy, mission statements, or organisational structure. However, having a strategy does not imply a separate operational budget for 'regional orientation and engagement'; only four universities claim to have earmarked financial resources. Besides the fact that universities may not single out their region as a specific target, their funds for innovation-related activities tend to be distributed across functional lines or by various organisational units of the university. Nonetheless, some degree of centralisation does occur at lower levels. Seven universities indicate that they run a separate organisational unit, or employ dedicated staff, devoted to 'regional orientation and engagement'. The size of that facility differs significantly; in one case it is a unit with less than six staff members, three have six to ten staff members, and the other three units employ more than ten each. Several are a 'Technology Transfer Office' (TTO) or 'Technological Innovation Centre' type of organisation, others have broader missions and names such as 'Center for Career Development' or 'Centre for Cooperation and Dialogue' clearly indicating that these units have objectives beyond that of the more traditional TTO.

Evidence-based management and monitoring of regional engagement activities and achievements requires the gathering of factual information. Most universities indicate that this is indeed common practice in their case, either for the university's annual report, for organisational strategy development, or accountability reporting for the benefit of external (national) stakeholders. In nine cases that information package included performance metrics, especially with regards to 'funding', 'education' or 'research'. RII-related information on 'infrastructure' appears to be much less amenable to measurement; only four universities apply metrics and quantitative performance indicators in this area.

Moving from collecting background information on a university's regional engagement capacity to gauging the applicability of our analytical framework, it is important to ascertain if that framework can capture a diversity of RII-relevant organisational missions, and main attributes of their 'RII portfolio': a complex mix of RII-relevant resources, infrastructures, pathways, programmes and collaborative arrangements. Are universities sufficiently capable to identify, describe and self-assess such a portfolio? In our survey we again framed this question within the broader context of their regional engagement activities. We posed the question "how is your university's focus 
on 'regional orientation and engagement' currently distributed (either in terms of invested resources, priority setting, or otherwise)?" We pre-selected the following categories to represent organisational missions: 'Teaching and training (students)', 'Human resource development (staff)'; 'Research, technological development and knowledge transfer', 'Entrepreneurship and enterprise development'; 'Physical infrastructure, knowledge infrastructure', 'Societal or cultural engagement and outreach'. These six categories correspond with our four RII portfolio domains. ${ }^{6}$ We asked the universities to express that distribution in terms of general levels; either 'none', 'low', 'average' or 'high'. The findings illustrate that this categorisation and differentiation was comprehensible and meaningful. Seven out of 11 universities that responded to this particular question indicated that 'Research, technological development and knowledge transfer' was in the 'high' category; only four universities did the same for the category 'Human resource development (staff)'. The overall results, displayed in Figure 4.1, showing a distribution significantly skewed towards 'Research, technological development and knowledge transfer' and 'Physical infrastructure, knowledge infrastructure', with 'Human resource development (staff)' and 'Societal or cultural engagement and outreach' on the other side of the spectrum. These disparities not only indicate major differences within these universities in targeting or priority setting, but also possibilities for developing systems that may create more equity and impetus across the various organisational missions in terms of promoting, supporting or accelerating regional engagement.

Such incentive or reward systems are used at four universities, which answered affirmatively the question "Does the university apply a reward or incentive system - for students or staff - with regards to their 'regional orientation and engagement' activities and achievements?". These dedicated systems apply to any of the above mission areas. One university, for example, applies incentives for infrastructure, with discounts for renting the spaces, both office and laboratory space. Another university distributes scholarships and awards for students.

It is revealing to find that out of 11 universities that collect data on their regional engagement, only four are actively promoting it with incentives and rewards. This finding suggests the presence of organisational cultures, poli-

6 The first two categories relate to the RII domain 'Education and human resources development', which is further discussed in Chapter 6. The third category relates to the RII domain 'Research, knowledge creation and technology transfer' (Chapter 7), while the fourth category aligns with the domain 'Support to enterprise development and entrepreneurship education' (Chapter 8). The last two categories relate to the domain 'Regional orientation, strategic development and knowledge infrastructure' (Chapter $5)$. 


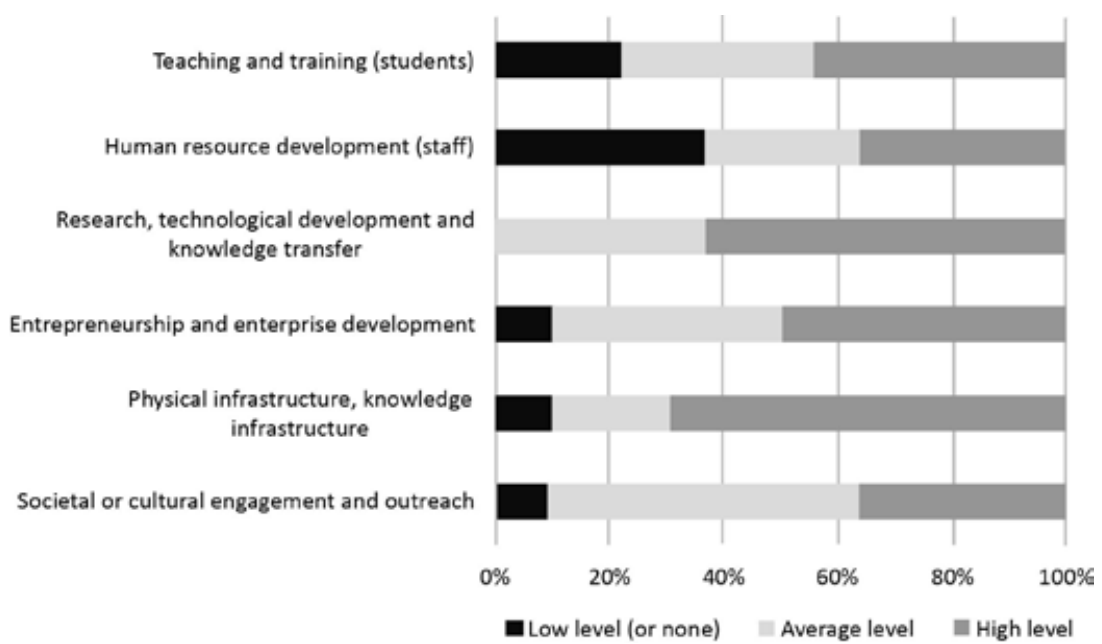

Note: $\quad$ One university answered 'none' for a domain, which was merged into the 'low' category to simplify comparability of scores across the domains.

\section{Figure 4.1 Level of regional engagement activities within universities} per domain (average score across universities)

cies, or other factors such as leadership commitments that prevent universities from applying such behaviour-influencing management tools. Touching on the issue of possible organisational obstacles, the survey's final question was "Are there specific area(s) in which your university faces major challenges with regard to developing or implementing 'regional orientation and engagement' initiatives or activities?" We specified seven domains, where such challenges may exist: 'Strategic priority setting at executive levels', 'Attracting or allocating dedicated funding', 'Attracting or allocating dedicated human resources', 'Creating general awareness among students or staff', 'Designing dedicated educational curricula or research programmes', 'Creating or upgrading infrastructures', and 'Engagement with local or regional partners'. We asked universities to respond to the question with a simple 'yes' or 'no'. Eleven universities responded and nine answered all questions. Figure 4.2 presents the aggregated findings. We found major differences as to where those challenges lie. Seven universities indicated that dedicated educational curricula or research programmes, focused on the local or regional environment, present them with problems. Dedicated funding also appears to be one of the headaches, as well as attracting or allocating human resources. Creating or upgrading infrastructures for regional engagement seems a relatively untroubled domain. Overall, we find that all these surveyed universities have developed 


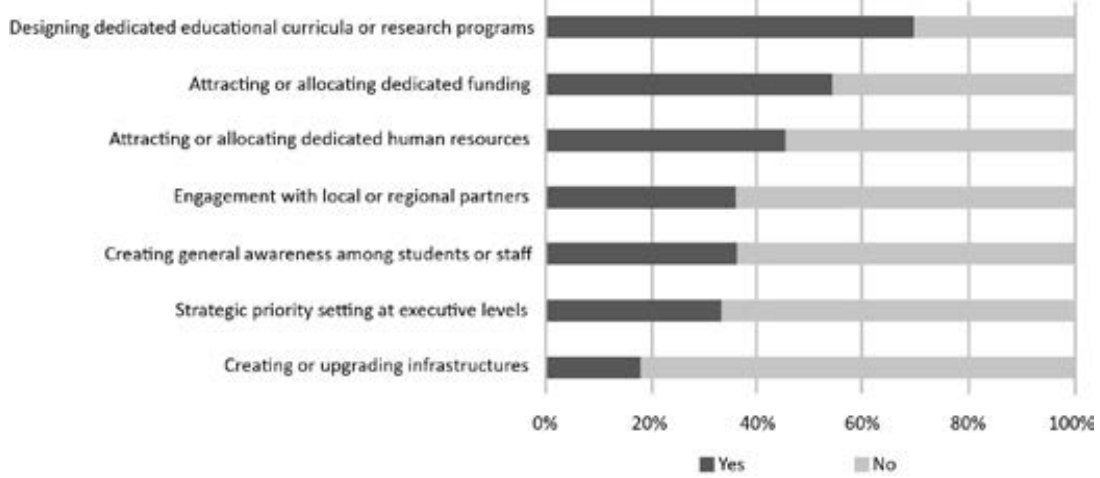

Figure 4.2 Existence of major challenges with regards to 'developing or implementing regional orientation and engagement initiatives or activities' (average score across universities)

organisational structures and facilities to reach out and interact with partners in their 'local' and 'regional' environment. All in all, the findings from this survey present interesting background information on their ability to undertake successful regional engagement, but also highlight important organisational issues concerning their current capacity to develop RII activities.

An interesting finding from this survey is the relatively low number of universities that have adopted dedicated incentive or reward systems to improve their regional orientation and engagement. Given the strong regional orientation of most universities one would expect to see more encouragement mechanisms in place for students and staff. Major challenges in specific areas, such as developing dedicated educational curricula or research programmes, suggest a need for further initiatives or policies to address such problems and obstacles. Implementing targeted incentives may be part of the solution or establishing more effective mechanisms to interact with municipal or regional stakeholders and leaders in order to calibrate regional engagement structures and practices. We return to the topic of incentives and funding instruments in Chapters 9 to 11 .

Each question invited additional commentary for supplementary information from universities. The survey's final 'open' question provided space for further general remarks. This feedback helped us to assess the general validity of our RII analytical model (depicted in Figure 2.1, section 2.1). This model assumes that a university's regional engagement 'goals' are largely determined by socio-economic or environmental 'needs, problems, issues' explicitly associated with the local or regional area. However, only a few universities 
acknowledge such strong ties with local or regional stakeholders, and a possible 'enforced' regional orientation.

In many other cases the involvement of those stakeholders tends to be implicit in so far as it is incorporated into the overall goal or broader 'ecosystem' strategies focusing on outreach and cooperation with society and industry in general. In one case, regional engagement is seen as part of the larger 'third mission' dedicated to creating public value via processes of open engagement. Another university has no specific central strategy on regional engagement, but has integrated it into educational and research activities of its individual staff members. Moreover, several universities indicate that goals are set at a broader level - with no specific reference to the local dimension - but several of them may nonetheless have a significant impact on the immediate geographical environment. Such 'global' universities do not restrict societal and economic impact strategies to the local region, but aim to create and maintain ecosystems that contribute to industrial and societal renewal irrespective of proximity - either locally, regionally, nationally or even globally.

Clearly our analytical model, which focuses exclusively on the immediate environment, falls short in terms of incorporating such broader spatial spill-over effects. The extended RII model in Figure 3.1 (section 3.1) provides an impression of how the local and regional is intertwined with the (inter) national environment.

\subsection{SELF-REPORTED RII INDICATORS}

The prescribed 'narrative with numbers' format offered the universities a wide range of possibilities for presenting their RII profile and describing their RII portfolio within the required general template (see Appendix A). We expected to find a lot of descriptive text, perhaps some data tables and graphs, but hopefully also some RII-specific statistical data. Both the 'narrative' and the 'numbers' parts of their self-appraisal report could contain references to specific empirical information on identifiable components of their 'RII potential' or 'RII performance' as shown in Box 4.1. Such information elements could be used, within an RII analysis framework, as a proxy measure (an 'indicator') to succinctly describe an RII profile. While a metrics-based 'quantitative indicator' would represent the volume or size of an element in a specific component, for example the number of students with internships in local firms, a non-metric 'qualitative' indicator would, for instance, refer to the occurrence (or absence) of a particular RII pathway within a university. 


\section{BOX 4.2 GENERAL CATEGORIES OF RII-RELATED INDICATORS}

Quantitative indicator - 'foreground' metrics-based information of RII potential or RII performance (e.g. percentage funding from local sources, number of TTO-generated results related to local SMEs, etc.); explicit reference to either the 'local' or 'regional' dimension of a university's portfolio or mission; includes specific numerical or statistical data;

Qualitative indicator - 'foreground' occurrence information on RII potential or RII performance (e.g. active participation in smart specialisation strategies; region-related targets in a strategic plan of a TTO, etc.); explicit reference to either the 'local' or 'regional' dimension of a university's portfolio or mission;

Other 'foreground' indicator - an integrated mix of quantitative data and qualitative information (or indicators with insufficient information to accurately determine their exact nature); explicit reference to either the 'local' or 'regional' dimension of a university's portfolio or mission;

'Background' indicator - no explicit reference to either the 'local' or 'regional' dimension of a university's portfolio or mission; directly or indirectly related to RII potential, RII performance or performance-enhancing support conditions (e.g. presence of RII relevant physical infrastructure in the area, relevant framework conditions such as impact-promoting university leadership, implementation of performance-based incentive systems, presence of a TTO or business accelerator facility or student entrepreneurship facilities, etc.).

Given the current lack of RII-specific quantitative indicators in general, one would expect to find a large share of qualitative indicators in their self-appraisal reporting, particularly in those components of their RII profile that are weakly developed in terms of in-house management information systems or measurability. To test this hypothesis we classified the references to such indicators into four categories: three types of 'foreground' indicators that specifically refer to the university's geographical area, and 'background' indicators with RII-relevant information of a more general nature (see Box 4.2 for more details). In our content analysis of the 20 university reports we identified 408 references in total, an average of 20 per case study. ${ }^{7}$ The summary statistics of the content analysis are presented in Table 4.2. The spread of references across

7 The exact number of references is difficult to determine for various reasons, but mainly because essential details are lacking in the text or the presence of ambiguous cases that could be allocated to more than one indicator category. 
Table 4.2 Distribution of RII indicator categories (20 case study self-appraisal reports)

\begin{tabular}{|c|c|c|c|c|c|}
\hline $\begin{array}{l}\text { Indicator category } \\
\text { RII portfolio domain }\end{array}$ & $\begin{array}{r}\text { References } \\
\text { (count) }\end{array}$ & $\begin{array}{r}\text { Quantitative } \\
\text { indicators } \\
\text { (\% of row } \\
\text { total) }\end{array}$ & $\begin{array}{r}\text { Qualitative } \\
\text { indicators } \\
\text { (\% of row } \\
\text { total) }\end{array}$ & $\begin{array}{r}\text { Other } \\
\text { foreground } \\
\text { indicators } \\
\text { (\% of row total) }\end{array}$ & $\begin{array}{r}\text { Background } \\
\text { indicators } \\
\text { (\% of row } \\
\text { total) }\end{array}$ \\
\hline $\begin{array}{l}\text { Regional orientation, } \\
\text { strategic development } \\
\text { and knowledge } \\
\text { infrastructure }\end{array}$ & 146 & $8 \%$ & $47 \%$ & $13 \%$ & $32 \%$ \\
\hline $\begin{array}{l}\text { Education and } \\
\text { human resources } \\
\text { development }\end{array}$ & 105 & $13 \%$ & $17 \%$ & $9 \%$ & $61 \%$ \\
\hline $\begin{array}{l}\text { Research, knowledge } \\
\text { creation and } \\
\text { technology transfer }\end{array}$ & 77 & $9 \%$ & $25 \%$ & $4 \%$ & $62 \%$ \\
\hline $\begin{array}{l}\text { Support to enterprise } \\
\text { development and } \\
\text { entrepreneurship } \\
\text { education }\end{array}$ & 75 & $7 \%$ & $25 \%$ & $0 \%$ & $68 \%$ \\
\hline Total & 403 & $9 \%$ & $31 \%$ & $8 \%$ & $52 \%$ \\
\hline
\end{tabular}

the four categories shows a dominance of 'background' indicators $(52 \%$ of all references) and qualitative 'foreground' indicators (31\%), while only $17 \%$ can be described as quantitative. Clearly, the 'narratives' dominate the 'numbers' in these self-appraisal reports.

Qualitative indicators are referred to relatively often when universities describe their activities in the 'Regional orientation, strategic development and knowledge infrastructure' domain of the RII profile; almost half (47\%) were qualitative indicators that implied a specific geographical impact. The most common qualitative indicators, occurring more than ten times, were 'cooperation with local/regional authorities', 'participation in regional clusters' or incubators and organisational changes to increase RII (such as 'changes to research portfolios, curricula or human resource policies'). Just $32 \%$ of the indicators were background indicators, far less than in the other three domains where the regional dimension is less explicit. Frequently occurring is the "commitment of the university to strategic regional partnerships', often formalised in a mission statement, as well as a 'commitment to solve regional skills shortages'. Finally, several quantitative indicators are also identified, such as the 'value of grants from regional organisations' and '\% of (regional) external board members in the university governing bodies'. Several indicators related to origin and des- 
tination of students were included in this section of the self-appraisal reports, despite it being an issue more relevant for the 'Education and human resources development' section. Content analysis of that section identifies more than 100 references across 25 indicators. Compared with the regional dimension, there was a greater proportion of background indicators (61\%). Some of the most common were on building entrepreneurial mind-sets among students, problem-based learning and recruitment rates (i.e. the percentage of students finding a job within 12 months). As for quantitative indicators (representing a $13 \%$ share of all indicators in this domain), most were related to the origin of students and lifelong learning; others were the 'number of internships' or 'percentage of students following work based courses'.

With regard to the 'Research, technological development and knowledge transfer' sections in the self-appraisal reports, almost two-thirds of the 77 observed cases are 'background' indicators. The vast majority relate to RII potential. Several indicators belong to facilities, infrastructures, and activities within component 3 in Box 4.1: 'Resources, general facilities and/ or incentive systems within the university'. Given the low numbers of references we decided to group several of those indicators into an aggregate-level 'composite' indicator that represents a 'structural' feature of the university's RII profile. The most prominent of those composite indicators, with 15 references, can be described as 'Dedicated facilities and infrastructures within the university for transfer and commercialisation'. This includes the existence of entrepreneurship centres, technology transfer offices, business incubators, and business accelerators. Three other composite indicators with more than five references are: 'Engagement or cooperation with industry and/or public sector networks' which may involve dedicated organisational consortia but also joint meeting platforms; 'Dedicated facilities and infrastructures within the university for internal processes' covering support offices, clubs and social platforms, and committees; 'General facilities and infrastructures outside the university', comprising science parks, shared laboratory space, co-working and co-creation spaces, and meeting platforms. Three of those composite indicators also appear in the list of 'qualitative' indicators, where the connections to local or regional users or partners are now quite explicit: 'Engagement or cooperation with industry and/or public sector partner networks'; 'Dedicated facilities and infrastructures within the university for transfer and commercialisation'; 'General facilities and infrastructures outside the university'. Three 'quantitative' indicators are mentioned more than once: 'Size of business sector oriented spaces (co-working and co-creation spaces, lab space, office space)'; 'Amount or share of funding from external local or regional sources'; and 'Number of research contracts, projects, post-doc positions, and/or partners in a local or regional partner'. 
As for the next section in the reports, on the RII domain 'Support to enterprise development and entrepreneurship education', here we identified 75 references, with a $68 \%$ share of 'background indicators'. There is a large similarity with the list of indicators mentioned in the 'Research, knowledge creation and technology transfer' domain, which is not surprising in view of the close organisational connections between both domains. By far the most frequently composite indicator referred to is, again, 'Dedicated facilities and infrastructures within university for transfer and commercialisation' with 20 references, many of which relate to entrepreneurship centres and dedicated education. The runner-up is the qualitative indicator 'Engagement or cooperation with industry and/or public sector partner networks in the region' (seven references), while the background indicator 'Dedicated facilities and infrastructures within university for internal processes' is mentioned five times. The latter indicator covers specialised support offices, specific clubs and social platforms, or special events such as student entrepreneurship competitions. Only three quantitative indicators are mentioned in all case study reports, each indicator only once. One of those three would almost certainly qualify as a high-profile 'key performance indicator' for any RII aspiring university: 'Number of jobs and amount of revenues created by spin-offs supported by the university's region-oriented business incubator'. Surprisingly, only one out of the 20 universities mentions this indicator explicitly. Section 9.1 discusses possible reasons for including or excluding particular indicators. 